\title{
Existence of periodic solutions for impulsive evolution equations in ordered Banach spaces
}

Huanhuan Zhang ${ }^{1,2^{*}}$, Yongxiang $\mathrm{Li}^{1}$ and Qiang $\mathrm{Li}^{1}$

"Correspondence:

sqhh1004@163.com

'Department of Mathematics,

Northwest Normal University,

Lanzhou, 730070, People's Republic of China

${ }^{2}$ School of Mathematics, Computer Science Institute, Northwest

University for Nationalities, Lanzhou, 730030, People's Republic of China

\begin{abstract}
In this paper, we use the perturbation method and the mixed monotone iterative technique to discuss the existence of periodic solutions for impulsive evolution equations in ordered Banach spaces. Under impulsive functions satisfying broader monotone conditions and without assumption that the lower and upper solutions exist, we obtain the existence results of $\omega$-periodic mild solutions. Moreover, an application is given to illustrate our theoretical results.
\end{abstract}

MSC: 35B10; 47H05

Keywords: semilinear impulsive evolution equation; upper and lower solutions; monotone iterative technique; periodic boundary value problems; $C_{0}$-semigroup

\section{Introduction}

In this paper, by using the perturbation method and the monotone iterative technique, we discuss the periodic solutions for the impulsive evolution equation

$$
\left\{\begin{array}{l}
u^{\prime}(t)+A u(t)=f(t, u(t)), \quad t \geq 0, t \neq t_{k}, \\
\left.\Delta u\right|_{t=t_{k}}=I_{k}\left(u\left(t_{k}\right)\right), \quad k \in \mathbf{N},
\end{array}\right.
$$

in an ordered Banach space $X$, where $A: D(A) \subset X \rightarrow X$ is a closed linear operator and $-A$ generates a positive $C_{0}$-semigroup $T(t)(t \geq 0)$ in $X ; f:[0,+\infty) \times X \rightarrow X$ is a continuous function and $f$ is $\omega$-periodic about $t$. $J=[0, \omega], \omega$ is a constant; $0<t_{1}<t_{2}<\cdots<t_{p}<\omega$. $I_{k}: X \rightarrow X(k=1,2, \ldots, p)$ are impulsive functions. $\left.\Delta u\right|_{t=t_{k}}=u\left(t_{k}^{+}\right)-u\left(t_{k}^{-}\right)$denotes the jump of $u(t)$ at $t=t_{k}$, where $u\left(t_{k}^{+}\right), u\left(t_{k}^{-}\right)$represent the right and left limits of $u(t)$ at $t=t_{k}(k \in \mathbf{N})$, respectively.

Obviously, the periodic problem of impulsive evolution equation (1.1) is equal to the periodic boundary value problem of impulsive evolution equation (IPBVP) in $J$,

$$
\left\{\begin{array}{l}
u^{\prime}(t)+A u(t)=f(t, u(t)), \quad t \in J, t \neq t_{k}, \\
\left.\Delta u\right|_{t=t_{k}}=I_{k}\left(u\left(t_{k}\right)\right), \quad k=1,2, \ldots, p, \\
u(0)=u(\omega) .
\end{array}\right.
$$

The study of impulsive differential equations is a new and important branch of differential equation theory for studying evolution processes of real life phenomena not only in

(c) 2015 Zhang et al. This article is distributed under the terms of the Creative Commons Attribution 4.0 International License (http://creativecommons.org/licenses/by/4.0/), which permits unrestricted use, distribution, and reproduction in any medium, provided you give appropriate credit to the original author(s) and the source, provide a link to the Creative Commons license, and indicate if changes were made. 
natural sciences but also in social sciences such as climate, food supplement, insecticide population, sustainable development that are subjected to sudden changes at certain instants. The theory of impulsive differential equations has been emerging as an important area of investigation in the last few decades; see the monographs of Lakshmikantham $e t$ al. [1], Benchohra et al. [2] and the papers of Chen [3], Li and Liu [4], Yang [5] and Lan [6], where numerous properties of their solutions are studied and detailed bibliographies are given. Consequently, some basic results on impulsive differential equations have been obtained and the applications of the theory of impulsive differential equations to different areas have been considered by many authors, see [4, 7-19] and the references therein.

The monotone iterative method based on lower and upper solutions is an effective and flexible mechanism. This technique is that, for the considered problem, starting from a pair of ordered lower and upper solutions, one constructs two monotone sequences such that they uniformly converge to the extremal solutions between the lower and upper solutions. By using the method of lower and upper solutions and the monotone iterative technique, Du and Lakshmikantham [20], Sun and Zhao [21] studied the existence of solutions to initial value problem of ordinary differential equation without impulse. Later on, Guo and Liu [22], Li and Liu [4] developed the monotone iterative method for impulsive integro-differential equations. Wang and Wang [23] investigated monotone iterative techniques for abstract semilinear evolution equations. Under the condition that the impulsive function is monotone increasing on the order interval, Chen and $\mathrm{Mu}$ [24] and Chen and Li [25] discussed the impulsive evolution equations with classical initial conditions.

Luo et al. [15] established a monotone iterative method for the antiperiodic boundary value problem of the first-order impulsive ordinary differential equations

$$
\left\{\begin{array}{l}
u^{\prime}(t)=f(t, u(t)), \quad t \in J, t \neq t_{k}, \\
\left.\Delta u\right|_{t=t_{k}}=I_{k}\left(u\left(t_{k}\right)\right), \quad k=1,2, \ldots, p, \\
u(0)=-u(\omega),
\end{array}\right.
$$

where the impulsive functions $I_{k}(k=1,2, \ldots, p)$ are nondecreasing. By applying the lower and upper solution method and the monotone iterative technique, the author obtained the existence of solutions for problem (1.3).

Ahmad and Nieto [26] applied the method of quasilinearization to obtain monotone sequences of approximate solutions converging uniformly and quadratically to the unique solution of the following impulsive anti-periodic problem:

$$
\left\{\begin{array}{l}
u^{\prime}(t)=g(t, x(t), x(w(t))), \quad t \in J=[0, T], t \neq t_{k}, t_{k} \in(0, T) \\
\Delta x\left(t_{k}\right)=I_{k}\left(x\left(t_{k}\right)\right), \quad k=1,2, \ldots, p \\
x(0)=-x(T) \\
x(t)=x(0), \quad t \in[-r, 0] .
\end{array}\right.
$$

Suppose that impulsive functions $I_{k}(k=1,2, \ldots, p)$ satisfied $-1 \leq I_{k}^{\prime}(\cdot) \leq 0$ with $I_{k}^{\prime \prime}(\cdot) \geq 0$.

Recently, Chen [3] discussed the existence of solutions to the impulsive periodic boundary value problem in an ordered Banach space $X$,

$$
\left\{\begin{array}{l}
u^{\prime}(t)=f(t, u(t), u(t)), \quad t \in J, t \neq t_{k}, \\
\left.\Delta u\right|_{t=t_{k}}=I_{k}\left(u\left(t_{k}\right), u\left(t_{k}\right)\right), \quad k=1,2, \ldots, p, \\
u(0)=u(\omega) .
\end{array}\right.
$$


Assume that problem (1.4) has coupled lower and upper $L$-quasisolutions $v_{0}$ and $w_{0}$ with $v_{0} \leq w_{0}$. Suppose that impulsive functions $I_{k}(k=1,2, \ldots, p)$ are satisfied

$$
I_{k}\left(u_{1}, v_{1}\right) \leq I_{k}\left(u_{2}, v_{2}\right), \quad k=1,2, \ldots, p
$$

for any $t \in J$ and $v_{0}(t) \leq u_{1} \leq u_{2} \leq w_{0}(t), v_{0}(t) \leq v_{2} \leq v_{1} \leq w_{0}(t)$.

Shao and Zhang [27] investigated the periodic solutions for the impulsive evolution equation

$$
\left\{\begin{array}{l}
u^{\prime}(t)+A u(t)=f(t, u(t)), \quad t \geq 0, t \neq t_{k} \\
\left.\Delta u\right|_{t=t_{k}}=I_{k}\left(u\left(t_{k}\right)\right), \quad k \in \mathbf{N}
\end{array}\right.
$$

where the impulsive functions satisfied the monotone condition $\forall t \in[0, \omega], v_{0}(t) \leq x_{1} \leq$ $x_{2} \leq w_{0}(t)$

$$
I_{k}\left(x_{1}\right) \leq I_{k}\left(x_{2}\right), \quad k=1,2, \ldots, p .
$$

In this paper, we consider the existence of $\omega$-periodic mild solutions for the impulsive evolution equation (1.2) by means of the perturbation method and the mixed monotone iterative technique. In the previous results in the related literature, the impulsive functions were considered as nondecreasing functions, which were not easy to satisfy. To our knowledge, there are very few papers to study the periodic boundary value problem of impulsive evolution equation under the impulsive functions satisfying quasimonotonicity. In this paper, we assume that the impulsive functions satisfy quasimonotonicity, which will compensate for the lack in this area. Applying $A=0$, our results improve and extend the evolution equations without impulse and some relevant results in ordinary differential equations.

\section{Preliminaries}

Let $X$ be a Banach space, $A: D(A) \subset X \rightarrow X$ be a closed linear operator and $-A$ generate a $C_{0}$-semigroup $T(t)(t \geq 0)$ in $X$. Then there exist constants $M>0$ and $v \in \mathbf{R}$ such that

$$
\begin{aligned}
& \|T(t)\| \leq M e^{v t}, \quad t \geq 0, \\
& \nu_{0}=\inf \left\{v \in \mathbf{R} \mid \exists M>0,\|T(t)\| \leq M e^{v t}, \forall t \geq 0\right\}
\end{aligned}
$$

and $v_{0}$ can also be expressed by $v_{0}=\lim \sup _{t \rightarrow+\infty} \frac{\ln \|T(t)\|}{t}$, then $v_{0}$ is called a growth index of the $C_{0}$-semigroup $T(t)(t \geq 0)$. If $v_{0}<0$, then $T(t)(t \geq 0)$ is called an exponentially stable $C_{0}$-semigroup.

Let $T(t)(t \geq 0)$ be an exponentially stable $C_{0}$-semigroup, for $\forall v \in\left(0,\left|v_{0}\right|\right)$, by the definition of $v_{0}$, we have $\exists M \geq 1$,

$$
\|T(t)\| \leq M e^{-v t}, \quad t \geq 0
$$

We define an equivalent norm in $X$ by

$$
|x|=\sup _{t \geq 0}\left\|e^{v t} T(t) x\right\|
$$


then $\|x\| \leq|x| \leq M\|x\|$. Respectively, $|T(t)|$ is the norm of the operator $T(t)$ in space $(X,|\cdot|)$. By $(2.2)$, we have

$$
|T(t)| \leq e^{-v t}
$$

and $|T(\omega)| \leq e^{-v \omega}<1$.

Lemma 2.1 [28] Let $T(t)(t \geq 0)$ be an exponentially stable $C_{0}$-semigroup, then the operator $I-T(\omega)$ has a bounded inverse operator $(I-T(\omega))^{-1}$ and satisfies the inequality

$$
\left|(I-T(w))^{-1}\right| \leq \frac{1}{1-e^{-v \omega}} .
$$

Let $X$ be an ordered Banach space with the norm $\|\cdot\|$ and partial order ' $\leq$ ', whose positive cone $K=\{x \in X \mid x \geq \theta\}$ is normal with normal constant $N . J=[0, \omega], \omega$ is a constant. Let $C(J, X)$ denote the Banach space of all continuous $X$-value functions on interval $J$ with the norm $\|u\|_{C}=\max _{t \in J}\|u(t)\|$. Then $C(J, X)$ is an ordered Banach space induced by the convex cone $K_{C}=\{u \in C(J, X) \mid u(t) \geq 0, t \in J\}$, and $K_{C}$ is also a normal cone.

Let $J^{\prime}=J \backslash\left\{t_{1}, t_{2}, \ldots, t_{p}\right\}, J^{\prime \prime}=J \backslash\left\{0, t_{1}, t_{2}, \ldots, t_{p}\right\}$. Let $J_{1}=\left[t_{0}, t_{1}\right], J_{k}=\left(t_{k-1}, t_{k}\right], k=$ $2,3, \ldots, p+1$, where $t_{0}=0, t_{p+1}=\omega$. Evidently, $P C(J, X)=\{u: J \rightarrow X \mid u(t)$ is continuous in $J^{\prime}$ and left continuous at $t_{k}$, and $u\left(t_{k}^{+}\right)$exists, $\left.k=1,2, \ldots, p\right\} . P C(J, X)$ is a Banach space with the norm $\|\cdot\|_{P C}=\sup _{t \in J}\|u(t)\|$. Evidently, $P C(J, X)$ is also an ordered Banach space with the partial order ' $\leq$ ' induced by the positive cone $K_{P C}=\{u \in P C(J, X) \mid u(t) \geq \theta, t \in J\} . K_{P C}$ is normal with the same normal constant $N$. For $v, w \in P C(J, X)$ with $v \leq w$, we use $[v, w]$ to denote the order interval $\{u \in P C(J, X) \mid v \leq u \leq w\}$ in $P C(J, X)$, and $[v(t), w(t)]$ to denote the order interval $\{u \in X \mid v(t) \leq u(t) \leq w(t), t \in J\}$ in $X$. We use $X_{1}$ to denote the Banach space $D(A)$ with the graph norm $\|\cdot\|_{1}=\|\cdot\|+\|A \cdot\|$.

Definition 2.2 If functions $v_{0} \in P C(J, X) \cap C^{1}\left(J^{\prime \prime}, X\right) \cap C\left(J^{\prime}, X_{1}\right)$ satisfy

$$
\left\{\begin{array}{l}
v_{0}^{\prime}(t)+A v_{0}(t) \leq f\left(t, v_{0}(t)\right), \quad t \in J, t \neq t_{k} \\
\left.\Delta v_{0}\right|_{t=t_{k}} \leq I_{k}\left(v_{0}\left(t_{k}\right)\right), \quad k=1,2, \ldots, p \\
v_{0}(0) \leq v_{0}(\omega)
\end{array}\right.
$$

we call $v_{0}$ a lower solution of IPBVP (1.2); if all the inequalities of (2.5) are inverse, we call it an upper solution of IPBVP (1.2).

\section{Linear impulsive evolution equation}

Let $I_{0}=\left[t_{0}, T\right]$. Denote by $C\left(I_{0}, X\right)$ the Banach space of all continuous $X$-value functions on interval $I_{0}$ with the norm $\|u\|_{C}=\max _{t \in I_{0}}\|u(t)\|$. It is well known [29] that for any $x_{0} \in D(A)$ and $h \in C^{1}\left(I_{0}, X\right)$, the initial value problem (IVP) of linear evolution equation

$$
\left\{\begin{array}{l}
u^{\prime}(t)+A u(t)=h(t), \quad t \in I_{0} \\
u\left(t_{0}\right)=x_{0}
\end{array}\right.
$$

has a unique classical solution $u \in C^{1}\left(I_{0}, X\right) \cap C\left(I_{0}, X_{1}\right)$ expressed by

$$
u(t)=T\left(t-t_{0}\right) x_{0}+\int_{t_{0}}^{t} T(t-s) h(s) d s, \quad t \in I_{0} .
$$


If $x_{0} \in X$ and $h \in C\left(I_{0}, X\right)$, the function $u$ given by (3.2) belongs to $C\left(I_{0}, X\right)$. We call it a mild solution of $\operatorname{IVP}(3.1)$. For any $h \in P C(J, X)$, we consider the periodic boundary value problem of linear impulsive evolution equation (LIPBVP) in $X$,

$$
\left\{\begin{array}{l}
u^{\prime}(t)+A u(t)=h(t), \quad t \in J, t \neq t_{k}, \\
\left.\Delta u\right|_{t=t_{k}}+a_{k} u\left(t_{k}\right)=e_{k}, \quad k=1,2, \ldots, p, \\
u(0)=u(\omega),
\end{array}\right.
$$

where $a_{k}$ is constant, $e_{k} \in X, k=1,2, \ldots, p$.

Theorem 3.1 Let $X$ be a Banach space, - A generate an exponentially stable $C_{0}$-semigroup $T(t)(t \geq 0)$ in $X$ and $v_{0}$ be a growth index of the semigroup $T(t)$. If $\frac{1}{\omega} \sum_{k=1}^{p} \ln \left(1-a_{k}\right)<-v_{0}$, for any $h \in P C(J, X), a_{k}<1$ is constant and $e_{k} \in X, k=1,2, \ldots, p, L I P B V P(3.3)$ has a unique mild solution $u \in P C(J, X)$ given by

$$
\begin{aligned}
u(t)= & \prod_{k: 0<t_{k}<t}\left(1-a_{k}\right) T(t) B_{1}(h)+\sum_{k: 0<t_{k}<t} \prod_{i: t_{k} \leq t_{i}<t}\left(1-a_{i}\right) \int_{t_{k-1}}^{t_{k}} T(t-s) h(s) d s \\
& +\int_{t_{j}}^{t} T(t-s) h(s) d s+\sum_{k: 0<t_{k}<t_{j} i: t_{k}<t_{i}<t} \prod\left(1-a_{i}\right) T\left(t-t_{k}\right) e_{k}+T\left(t-t_{j}\right) e_{j} \\
= & Q_{1}(h),
\end{aligned}
$$

where $t_{j}<t(j=0,1,2, \ldots, p)$ is the nearest point of $t, j=\max \left\{k \mid 0<t_{k}<t\right\}$ and

$$
\begin{aligned}
B_{1}(h)= & {\left[I-\prod_{k=1}^{p}\left(1-a_{k}\right) T(\omega)\right]^{-1}\left[\sum_{k=1}^{p} \prod_{i=k}^{p}\left(1-a_{i}\right) \int_{t_{k-1}}^{t_{k}} T(\omega-s) h(s) d s\right.} \\
& \left.+\int_{t_{p}}^{\omega} T(\omega-s) h(s) d s+\sum_{k=1}^{p-1} \prod_{i=k+1}^{p}\left(1-a_{i}\right) T\left(\omega-t_{k}\right) e_{k}+T\left(\omega-t_{p}\right) e_{p}\right] .
\end{aligned}
$$

Proof Let $J_{1}=\left[t_{0}, t_{1}\right], J_{k}=\left(t_{k-1}, t_{k}\right], k=2,3, \ldots, p+1$, where $t_{0}=0, t_{p+1}=\omega$. For any $h \in$ $P C(J, X)$, we first show that the initial value problem of linear impulsive evolution equation

$$
\left\{\begin{array}{l}
u^{\prime}(t)+A u(t)=h(t), \quad t \in J, t \neq t_{k}, \\
\left.\Delta u\right|_{t=t_{k}}+a_{k} u\left(t_{k}\right)=e_{k}, \quad k=1,2, \ldots, p, \\
u(0)=x_{0} .
\end{array}\right.
$$

Let $t \in J_{1}=\left[t_{0}, t_{1}\right]$, equation (3.5) is equivalent to the initial value problem of linear evolution equation

$$
\left\{\begin{array}{l}
u^{\prime}(t)+A u(t)=h(t), \quad t \in J_{1}, \\
u(0)=x_{0} .
\end{array}\right.
$$

Then (3.6) has a unique mild solution $u_{1} \in C\left(J_{1}, X\right)$ given by

$$
u_{1}(t)=T(t) x_{0}+\int_{0}^{t} T(t-s) h(s) d s
$$


Especially, we have

$$
u_{1}\left(t_{1}\right)=T\left(t_{1}\right) x_{0}+\int_{0}^{t_{1}} T\left(t_{1}-s\right) h(s) d s \in X .
$$

Let $t \in J_{2}=\left(t_{1}, t_{2}\right]$, equation (3.5) is equivalent to the initial value problem of linear evolution equation

$$
\left\{\begin{array}{l}
u^{\prime}(t)+A u(t)=h(t), \quad t \in J_{2} \\
u\left(t_{1}^{+}\right)=u_{1}\left(t_{1}\right)-a_{1} u_{1}\left(t_{1}\right)+e_{1}
\end{array}\right.
$$

Combining with (3.7), then (3.8) has a unique mild solution $u_{2} \in C\left(J_{2}, X\right)$ expressed by

$$
\begin{aligned}
u_{2}(t) & =T\left(t-t_{1}\right)\left[\left(1-a_{1}\right) u_{1}\left(t_{1}\right)+e_{1}\right]+\int_{t_{1}}^{t} T(t-s) h(s) d s \\
& =T\left(t-t_{1}\right)\left[\left(1-a_{1}\right)\left(T\left(t_{1}\right) x_{0}+\int_{0}^{t_{1}} T\left(t_{1}-s\right) h(s) d s\right)+e_{1}\right]+\int_{t_{1}}^{t} T(t-s) h(s) d s \\
& =\left(1-a_{1}\right) T(t) x_{0}+\left(1-a_{1}\right) \int_{0}^{t_{1}} T(t-s) h(s) d s+\int_{t_{1}}^{t} T(t-s) h(s) d s+T\left(t-t_{1}\right) e_{1} .
\end{aligned}
$$

Particularly, we have

$$
u_{2}\left(t_{2}\right)=\left(1-a_{1}\right) T\left(t_{2}\right) x_{0}+\left(1-a_{1}\right) \int_{0}^{t_{1}} T\left(t_{2}-s\right) h(s) d s+\int_{t_{1}}^{t_{2}} T\left(t_{2}-s\right) h(s) d s+T\left(t_{2}-t_{1}\right) e_{1} .
$$

Similarly, let $t \in J_{k}=\left(t_{k-1}, t_{k}\right](k=3,4, \ldots, p+1)$, where $t_{p+1}=\omega$, equation (3.5) is equivalent to the initial value problem of linear evolution equation

$$
\left\{\begin{array}{l}
u^{\prime}(t)+A u(t)=h(t), \quad t \in J_{k}, \\
u\left(t_{k-1}^{+}\right)=\left(1-a_{k-1}\right) u\left(t_{k-1}\right)+e_{k-1}, \quad k=3,4, \ldots, p+1
\end{array}\right.
$$

Then (3.9) has a unique mild solution $u_{k} \in C\left(J_{k}, X\right)$ expressed by

$$
\begin{aligned}
u_{k}(t)= & T\left(t-t_{k-1}\right)\left[\left(1-a_{k-1}\right) u_{k-1}\left(t_{k-1}\right)+e_{k-1}\right]+\int_{t_{k-1}}^{t} T(t-s) h(s) d s \\
= & \prod_{k: 0<t_{k}<t}\left(1-a_{k}\right) T(t) x_{0}+\sum_{k: 0<t_{k}<t} \prod_{i: t_{k} \leq t_{i}<t}\left(1-a_{i}\right) \int_{t_{k-1}}^{t_{k}} T(t-s) h(s) d s \\
& +\int_{t_{j}}^{t} T(t-s) h(s) d s+\sum_{k: 0<t_{k}<t_{j}: i t_{k}<t_{i}<t}\left(1-a_{i}\right) T\left(t-t_{k}\right) e_{k}+T\left(t-t_{j}\right) e_{j},
\end{aligned}
$$

where $t_{j}<t(j=0,1,2, \ldots, p)$ is the nearest point of $t, j=\max \left\{k \mid 0<t_{k}<t\right\}$.

Let

$$
u(t)= \begin{cases}u_{1}(t), & t \in J_{1}, \\ u_{2}(t), & t \in J_{2}, \\ \cdots, & \\ u_{p+1}(t), & t \in J_{p+1} .\end{cases}
$$


Inversely, the function $u \in P C(J, X)$ defined by (3.10) is a unique mild solution of the initial value problem of linear evolution equation (3.5).

Next,we show that LIPBVP (3.3) has a unique mild solution $u \in P C(J, X)$ given by (3.4). If a function $u \in P C(J, X)$ defined by (3.10) is a solution of LIPBVP (3.3), then $x_{0}=u(\omega)$, namely

$$
\begin{aligned}
(I- & \left.\prod_{k=1}^{p}\left(1-a_{k}\right) T(\omega)\right) x_{0} \\
= & \sum_{k=1}^{p} \prod_{i=k}^{p}\left(1-a_{i}\right) \int_{t_{k-1}}^{t_{k}} T(\omega-s) h(s) d s+\int_{t_{p}}^{\omega} T(\omega-s) h(s) d s \\
& +\sum_{k=1}^{p-1} \prod_{i=k+1}^{p}\left(1-a_{i}\right) T\left(\omega-t_{k}\right) e_{k}+T\left(\omega-t_{p}\right) e_{p} .
\end{aligned}
$$

For $\forall v \in\left(0,-v_{0}\right)$, by Lemma 2.1, since $r\left(\prod_{k=1}^{p}\left(1-a_{k}\right) T(\omega)\right) \leq \prod_{k=1}^{p}\left|1-a_{k}\right| e^{-v \omega}$, and $\frac{1}{\omega} \sum_{k=1}^{p} \ln \left(1-a_{k}\right)<-v_{0}, a_{k}<1$, by the arbitrary of $v$, we have $r\left(\prod_{k=1}^{p}\left(1-a_{k}\right) T(\omega)\right) \leq$ $\prod_{k=1}^{p}\left(1-a_{k}\right) e^{v_{0} \omega}<1$, and $I-\prod_{k=1}^{p}\left(1-a_{k}\right) T(\omega)$ has a bounded inverse operator. From (3.11), we choose

$$
\begin{aligned}
x_{0}= & {\left[I-\prod_{k=1}^{p}\left(1-a_{k}\right) T(\omega)\right]^{-1}\left[\sum_{k=1}^{p} \prod_{i=k}^{p}\left(1-a_{i}\right) \int_{t_{k-1}}^{t_{k}} T(\omega-s) h(s) d s\right.} \\
& \left.+\int_{t_{p}}^{\omega} T(\omega-s) h(s) d s+\sum_{k=1}^{p-1} \prod_{i=k+1}^{p}\left(1-a_{i}\right) T\left(\omega-t_{k}\right) e_{k}+T\left(\omega-t_{p}\right) e_{p}\right] \\
\triangleq & B_{1}(h) .
\end{aligned}
$$

Combining (3.12) with (3.10), we obtain that the function $u(t) \in P C(J, X)$ given by (3.4) is a unique mild solution of LIBPVP (3.3) on $J$ and the operator $Q_{1}: P C(J, X) \rightarrow P C(J, X)$ is a continuous operator. So, the conclusion of Theorem 3.1 holds.

Remark 3.2 In Theorem 3.1, let $X$ be an ordered Banach space, $-A$ generate a positive $C_{0}$-semigroup $T(t)(t \geq 0)$ in $X$ and $v_{0}$ be a growth index of the semigroup $T(t)$. If $\frac{1}{\omega} \sum_{k=1}^{p} \ln \left(1-a_{k}\right)<-v_{0}$, for any $h \geq \theta, a_{k}<1$ is constant and $e_{k} \geq \theta, k=1,2, \ldots, p$, then the solution operator $Q_{1}$ of LIPBVP (3.3) is a positive operator.

\section{Proof of the main results}

Theorem 4.1 Let $X$ be an ordered Banach space, whose positive cone $K$ is normal, and $N_{0}$ be the normal constant. Let $A: D(A) \subset X \rightarrow X$ be a closed linear operator and $-A$ generate a compact and positive $C_{0}$-semigroup $T(t)(t \geq 0)$ in $X . f \in C(J \times X, X)$ is $\omega$-periodic about $t, I_{k} \in C(X, X), k=1,2, \ldots, p$. Assume that IPBVP (1.2) has lower and upper solutions $v_{0}$ and $w_{0}$ with $v_{0}(t) \leq w_{0}(t)(t \in J)$. Suppose that the following conditions are satisfied:

$\left(\mathrm{P}_{1}\right)$ There exists a constant $C \geq 0$ such that

$$
f\left(t, x_{2}\right)-f\left(t, x_{1}\right) \geq-C\left(x_{2}-x_{1}\right), \quad t \in J,
$$

for $\forall t \in J, v_{0}(t) \leq x_{1} \leq x_{2} \leq w_{0}(t)$. 
$\left(\mathrm{P}_{2}\right) \quad \exists 0 \leq N_{k}<1$, for $\forall t \in J, v_{0}(t) \leq x_{1} \leq x_{2} \leq w_{0}(t)$, impulsive functions $I_{k}$ satisfy

$$
I_{k}\left(x_{2}\right)-I_{k}\left(x_{1}\right) \geq-N_{k}\left(x_{2}-x_{1}\right), \quad k=1,2, \ldots, p .
$$

Then IPBVP (1.2) has minimal and maximal $\omega$-periodic mild solutions $\underline{u}$ and $\bar{u}$ between $v_{0}$ and $w_{0}$, which can be obtained by monotone iterative sequences starting from $v_{0}$ and $w_{0}$.

Proof Define $D=\left[v_{0}, w_{0}\right]$. For $\forall h \in D$, we consider the periodic boundary value problem of linear impulsive evolution equation (LIPBVP) in $X$,

$$
\left\{\begin{array}{l}
u^{\prime}(t)+A u(t)+C u(t)=f(t, h(t))+C h(t), \quad t \in J, t \neq t_{k}, \\
\left.\Delta u\right|_{t=t_{k}}+N_{k} u\left(t_{k}\right)=I_{k}\left(h\left(t_{k}\right)\right)+N_{k} h\left(t_{k}\right), \quad k=1,2, \ldots, p, \\
u(0)=u(\omega),
\end{array}\right.
$$

where $\forall t \in[0, \omega]$, define $t_{j}<t(j=0,1,2, \ldots, p)$ is the nearest point of $t, j=\max \left\{k \mid 0<t_{k}<\right.$ $t\}, f_{1}(t, x)=f(t, x)+C x$.

Let $C>v_{0},-(A+C I)$ generate an exponentially stable, compact and positive $C_{0}$-semigroup $S(t)=e^{-C t} T(t)(t \geq 0)$ in $X$, whose growth index is $-C+v_{0}$. Since $C>v_{0}, 0 \leq N_{k}<1$, so

$$
\frac{1}{\omega} \sum_{k=1}^{p} \ln \left(1-N_{k}\right)<C-v_{0}
$$

From Theorem 3.1, LIPBVP (4.1) has a unique mild solution $u \in P C(J, X)$ given by

$$
\begin{aligned}
u(t)= & \prod_{k: 0<t_{k}<t}\left(1-N_{k}\right) S(t) B_{2}(h)+\sum_{k: 0<t_{k}<t} \prod_{i: t_{k} \leq t_{i}<t}\left(1-N_{i}\right) \int_{t_{k-1}}^{t_{k}} S(t-s) f_{1}(s, h(s)) d s \\
& +\int_{t_{j}}^{t} S(t-s) f_{1}(s, h(s)) d s+\sum_{k: 0<t_{k}<t_{j}} \prod_{i: t_{k}<t_{i}<t}\left(1-N_{i}\right) S\left(t-t_{k}\right)\left(I_{k}\left(h\left(t_{k}\right)\right)+N_{k} h\left(t_{k}\right)\right) \\
& +S\left(t-t_{j}\right)\left(I_{j}\left(h\left(t_{j}\right)\right)+N_{j} h\left(t_{j}\right)\right) \triangleq Q_{2}(h),
\end{aligned}
$$

where

$$
\begin{aligned}
B_{2}(h) \triangleq & \left(I-\prod_{k=1}^{p}\left(1-N_{k}\right) S(\omega)\right)^{-1}\left[\sum_{k=1}^{p} \prod_{i=k}^{p}\left(1-N_{i}\right) \int_{t_{k-1}}^{t_{k}} S(\omega-s) f_{1}(s, h(s)) d s\right. \\
& +\int_{t_{p}}^{\omega} S(\omega-s) f_{1}(s, h(s)) d s+\sum_{k=1}^{p-1} \prod_{i=k+1}^{p}\left(1-N_{i}\right) S\left(\omega-t_{k}\right)\left(I_{k}\left(h\left(t_{k}\right)\right)+N_{k} h\left(t_{k}\right)\right) \\
& \left.+S\left(\omega-t_{p}\right)\left(I_{p}\left(h\left(t_{p}\right)\right)+N_{p} h\left(t_{p}\right)\right)\right]
\end{aligned}
$$

Since $f$ and $I_{k}$ are continuous, so $Q_{2}: D \rightarrow P C(J, X)$ is continuous.

Clearly, the $\omega$-periodic mild solutions of IPBVP (1.2) are equivalent to the fixed points of operator $Q_{2}$.

(i) We show $Q_{2}: D \rightarrow P C(J, X)$ is an increasing operator. 
In fact, for $\forall h_{1}, h_{2} \in D$ and $h_{1} \leq h_{2}$, from the assumptions $\left(\mathrm{P}_{1}\right)$ and $\left(\mathrm{P}_{2}\right)$, we have

$$
f_{1}\left(t, h_{1}(t)\right)=f\left(t, h_{1}(t)\right)+C h_{1}(t) \leq f\left(t, h_{2}(t)\right)+C h_{2}(t)=f_{1}\left(t, h_{2}(t)\right), \quad t \in J
$$

and

$$
I_{k}\left(h_{1}\left(t_{k}\right)\right)+N_{k} h_{1}\left(t_{k}\right) \leq I_{k}\left(h_{2}\left(t_{k}\right)\right)+N_{k} h_{2}\left(t_{k}\right), \quad k=1,2, \ldots, p .
$$

Since $S(t)$ is an exponentially stable and positive $C_{0}$-semigroup, combining this with (4.2), then $\left[I-\prod_{k=1}^{p}\left(1-N_{k}\right) S(\omega)\right]$ has a bounded inverse operator and it can be expressed that $\left(I-\prod_{k=1}^{p}\left(1-N_{k}\right) S(\omega)\right)^{-1}=\sum_{n=0}^{\infty}\left(\prod_{k=1}^{p}\left(1-N_{k}\right)\right)^{n} S(n \omega)$. Obviously, the operator $(I-$ $\left.\prod_{k=1}^{p}\left(1-N_{k}\right) S(\omega)\right)^{-1}$ is a positive operator. Hence, we have

$$
\begin{aligned}
& \sum_{k=1}^{p} \prod_{i=k}^{p}\left(1-N_{i}\right) \int_{t_{k-1}}^{t_{k}} S(\omega-s) f_{1}\left(s, h_{1}(s)\right) d s+\int_{t_{p}}^{\omega} S(\omega-s) f_{1}\left(s, h_{1}(s)\right) d s \\
& \quad \leq \sum_{k=1}^{p} \prod_{i=k}^{p}\left(1-N_{i}\right) \int_{t_{k-1}}^{t_{k}} S(\omega-s) f_{1}\left(s, h_{2}(s)\right) d s+\int_{t_{p}}^{\omega} S(\omega-s) f_{1}\left(s, h_{2}(s)\right) d s
\end{aligned}
$$

and

$$
\begin{aligned}
& \sum_{k=1}^{p-1} \prod_{i=k+1}^{p}\left(1-N_{i}\right) S\left(\omega-t_{k}\right)\left(I_{k}\left(h_{1}\left(t_{k}\right)\right)+N_{k} h_{1}\left(t_{k}\right)\right)+S\left(\omega-t_{p}\right)\left(I_{p}\left(h_{1}\left(t_{p}\right)\right)+N_{p} h_{1}\left(t_{p}\right)\right) \\
& \leq \sum_{k=1}^{p-1} \prod_{i=k+1}^{p}\left(1-N_{i}\right) S\left(\omega-t_{k}\right)\left(I_{k}\left(h_{2}\left(t_{k}\right)\right)+N_{k} h_{2}\left(t_{k}\right)\right) \\
& \quad+S\left(\omega-t_{p}\right)\left(I_{p}\left(h_{2}\left(t_{p}\right)\right)+N_{p} h_{2}\left(t_{p}\right)\right) .
\end{aligned}
$$

Namely, $B_{2}\left(h_{1}\right) \leq B_{2}\left(h_{2}\right)$. Thus we obtain the inequality $\prod_{k: 0<t_{k}<t}\left(1-N_{k}\right) S(t) B_{2}\left(h_{1}\right) \leq$ $\prod_{k: 0<t_{k}<t}\left(1-N_{k}\right) S(t) B_{2}\left(h_{2}\right)$. Combining this with (4.3), we have $Q_{2}\left(h_{1}\right) \leq Q_{2}\left(h_{2}\right)$.

(ii) We show $v_{0} \leq Q_{2}\left(v_{0}\right), Q_{2}\left(w_{0}\right) \leq w_{0}$.

Let

$$
\left\{\begin{array}{l}
v_{0}^{\prime}(t)+A v_{0}(t)+C v_{0}(t)=\bar{h}(t), \quad t \in J, t \neq t_{k} \\
\left.\Delta v_{0}\right|_{t=t_{k}}+N_{k} v_{0}\left(t_{k}\right)=\bar{e}_{k}, \quad k=1,2, \ldots, p \\
v_{0}(0)=v_{0}(\omega)
\end{array}\right.
$$

from the definition of $v_{0}$, we have

$$
\left\{\begin{array}{l}
\bar{h}(t) \leq f\left(t, v_{0}(t)\right)+C v_{0}(t), \quad t \in J, t \neq t_{k}, \\
\bar{e}_{k} \leq I_{k}\left(v_{0}\left(t_{k}\right)\right)+N_{k} v_{0}\left(t_{k}\right), \quad k=1,2, \ldots, p .
\end{array}\right.
$$

By Theorem 3.1, (4.4) and (4.5), we have

$$
\begin{aligned}
v_{0}(t)= & \prod_{k: 0<t_{k}<t}\left(1-N_{k}\right) S(t) B_{3}(\bar{h})+\sum_{k: 0<t_{k}<t} \prod_{i: t_{k} \leq t_{i}<t}\left(1-N_{i}\right) \int_{t_{k-1}}^{t_{k}} S(t-s) \bar{h}(s) d s \\
& +\int_{t_{j}}^{t} S(t-s) \bar{h}(s) d s+\sum_{k: 0<t_{k}<t_{j}: i t_{k}<t_{i}<t} \prod_{t}\left(1-N_{i}\right) S\left(t-t_{k}\right) \bar{e}_{k}+S\left(t-t_{j}\right) \bar{e}_{j}
\end{aligned}
$$




$$
\begin{aligned}
\leq & \prod_{k: 0<t_{k}<t}\left(1-N_{k}\right) S(t) B_{3}(\bar{h})+\sum_{k: 0<t_{k}<t} \prod_{i: t_{k} \leq t_{i}<t}\left(1-N_{j}\right) \int_{t_{k-1}}^{t_{k}} S(t-s) f_{1}\left(s, v_{0}(s)\right) d s \\
& +\int_{t_{j}}^{t} S(t-s) f_{1}\left(s, v_{0}(s)\right) d s \\
& +\sum_{k: 0<t_{k}<t_{j}} \prod_{i: t_{k}<t_{i}<t}\left(1-N_{i}\right) S\left(t-t_{k}\right)\left(I_{k}\left(v_{0}\left(t_{k}\right)\right)+N_{k} v_{0}\left(t_{k}\right)\right) \\
& +S\left(t-t_{j}\right)\left(I_{j}\left(v_{0}\left(t_{j}\right)\right)+N_{j} v_{0}\left(t_{j}\right)\right),
\end{aligned}
$$

where

$$
\begin{aligned}
B_{3}(\bar{h})= & \left(I-\prod_{k=1}^{p}\left(1-N_{k}\right) S(\omega)\right)^{-1}\left[\sum_{k=1}^{p} \prod_{i=k}^{p}\left(1-N_{i}\right) \int_{t_{k-1}}^{t_{k}} S(\omega-s) \bar{h}(s) d s\right. \\
& \left.+\int_{t_{p}}^{\omega} S(\omega-s) \bar{h}(s) d s+\sum_{k=1}^{p-1} \prod_{i=k+1}^{p}\left(1-N_{i}\right) S\left(\omega-t_{k}\right) \bar{e}_{k}+S\left(\omega-t_{p}\right) \bar{e}_{p}\right] .
\end{aligned}
$$

Particularly,

$$
\begin{aligned}
v_{0}(\omega) \leq & \prod_{k=1}^{p}\left(1-N_{k}\right) S(\omega) B_{3}(\bar{h})+\sum_{k=1}^{p} \prod_{i=k}^{p}\left(1-N_{i}\right) \int_{t_{k-1}}^{t_{k}} S(\omega-s) f_{1}\left(s, v_{0}(s)\right) d s \\
& +\int_{t_{p}}^{\omega} S(\omega-s) f_{1}\left(s, v_{0}(s)\right) d s \\
& +\sum_{k=1}^{p-1} \prod_{i=k+1}^{p}\left(1-N_{i}\right) S\left(\omega-t_{k}\right)\left(I_{k}\left(v_{0}\left(t_{k}\right)\right)+N_{k} v_{0}\left(t_{k}\right)\right) \\
& +S\left(\omega-t_{p}\right)\left(I_{p}\left(v_{0}\left(t_{p}\right)\right)+N_{p} v_{0}\left(t_{p}\right)\right) .
\end{aligned}
$$

By (4.4) and (4.6), then $v_{0}(0)=B_{3}(\bar{h})$. Combining $v_{0}(0) \leq v_{0}(\omega)$ with (4.7), we have

$$
\begin{aligned}
B_{3}(\bar{h}) \leq & \left(I-\prod_{k=1}^{p}\left(1-N_{k}\right) S(\omega)\right)^{-1}\left[\sum_{k=1}^{p} \prod_{i=k}^{p}\left(1-N_{i}\right) \int_{t_{k-1}}^{t_{k}} S(\omega-s) f_{1}\left(s, v_{0}(s)\right) d s\right. \\
& +\int_{t_{p}}^{\omega} S(\omega-s) f_{1}\left(s, v_{0}(s)\right) d s+\sum_{k=1}^{p-1} \prod_{i=k+1}^{p}\left(1-N_{i}\right) S\left(\omega-t_{k}\right)\left(I_{k}\left(v_{0}\left(t_{k}\right)\right)+N_{k} v_{0}\left(t_{k}\right)\right) \\
& \left.+S\left(\omega-t_{p}\right)\left(I_{p}\left(v_{0}\left(t_{p}\right)\right)+N_{p} v_{0}\left(t_{p}\right)\right)\right] \\
= & B_{2}\left(v_{0}\right) .
\end{aligned}
$$

On the other hand, from (4.3), then

$$
\begin{aligned}
Q_{2}\left(v_{0}\right)(t)= & \prod_{k: 0<t_{k}<t}\left(1-N_{k}\right) S(t) B_{2}\left(v_{0}\right)+\sum_{k: 0<t_{k}<t} \prod_{i: t_{k} \leq t_{i}<t}\left(1-N_{i}\right) \int_{t_{k-1}}^{t_{k}} S(t-s) f_{1}\left(s, v_{0}(s)\right) d s \\
& +\int_{t_{j}}^{t} S(t-s) f_{1}\left(s, v_{0}(s)\right) d s
\end{aligned}
$$




$$
\begin{aligned}
& +\sum_{k: 0<t_{k}<t_{j} i: t_{k}<t_{i}<t}\left(1-N_{i}\right) S\left(t-t_{k}\right)\left(I_{k}\left(v_{0}\left(t_{k}\right)\right)+N_{k} v_{0}\left(t_{i}\right)\right) \\
& +S\left(t-t_{j}\right)\left(I_{j}\left(v_{0}\left(t_{j}\right)\right)+N_{j} v_{0}\left(t_{j}\right)\right) .
\end{aligned}
$$

By (4.6) and (4.8), we have

$$
Q_{2}\left(v_{0}\right)(t)-v_{0}(t) \geq \prod_{k: 0<t_{k}<t}\left(1-N_{k}\right) S(t)\left(B_{2}\left(v_{0}\right)-B_{3}(\bar{h})\right) \geq \theta,
$$

namely $v_{0}(t) \leq Q_{2}\left(v_{0}\right)(t)$. Similarly, it can be shown that $Q_{2}\left(w_{0}\right)(t) \leq w_{0}(t)$. Therefore, $Q_{2}$ : $\left[v_{0}, w_{0}\right] \rightarrow\left[v_{0}, w_{0}\right]$ is a continuously increasing operator.

(iii) Next, we will prove that the operator $Q_{2}$ has fixed points on $\left[v_{0}, w_{0}\right]$.

Now, we define two sequences $\left\{v_{n}\right\}$ and $\left\{w_{n}\right\}$ by the iterative scheme

$$
v_{n}=Q_{2}\left(v_{n-1}\right), \quad w_{n}=Q_{2}\left(w_{n-1}\right), \quad n=1,2, \ldots
$$

Then from the monotonicity of operator $Q_{2}$ it follows that

$$
v_{0} \leq v_{1} \leq v_{2} \leq \cdots \leq v_{n} \leq \cdots \leq w_{n} \leq \cdots \leq w_{2} \leq w_{1} \leq w_{0}
$$

Next, we prove that $\left\{v_{n}\right\}$ and $\left\{w_{n}\right\}$ are convergent in $J$. Let $G=\left\{v_{n} \mid n \in \mathbf{N}\right\}, G_{0}=\left\{v_{n-1} \mid n \in\right.$ $\mathbf{N}\}$, then $G_{0}=\left\{v_{0}\right\} \cup G$ and $G=Q_{2}\left(G_{0}\right)$. For any $v_{n-1} \in G_{0}$, let

$$
\begin{aligned}
W\left(v_{n-1}\right)(t)= & \sum_{k: 0<t_{k}<t i: t_{k} \leq t_{i}<t}\left(1-N_{i}\right) \int_{t_{k-1}}^{t_{k}} S(t-s) f_{1}\left(s, v_{n-1}(s)\right) d s \\
& +\int_{t_{j}}^{t} S(t-s) f_{1}\left(s, v_{n-1}(s)\right) d s \\
& +\sum_{k: 0<t_{k}<t_{j}: i_{k}<t_{i}<t}\left(1-N_{i}\right) S\left(t-t_{k}\right)\left(I_{k}\left(v_{n-1}\left(t_{k}\right)\right)+N_{k} v_{n-1}\left(t_{k}\right)\right) \\
& +S\left(t-t_{j}\right)\left(I_{j}\left(v_{n-1}\left(t_{j}\right)\right)+N_{j} v_{n-1}\left(t_{j}\right)\right),
\end{aligned}
$$

then $Q_{2}\left(v_{n-1}\right)(t)=\prod_{k: 0<t_{k}<t}\left(1-N_{k}\right) S(t) B_{2}\left(v_{n-1}\right)+W\left(v_{n-1}\right)(t)$. First, we will prove that for any $0<t<\omega, Y(t) \stackrel{\text { def }}{=}\left\{W\left(v_{n-1}\right)(t) \mid v_{n-1} \in G_{0}\right\}$ is relatively compact in $X$. Let $0<\epsilon<t$, $\bar{j}=\max \left\{k \mid 0<t_{k}<t-\epsilon\right\}$ and

$$
\begin{aligned}
W_{\epsilon}\left(v_{n-1}\right)(t)= & \sum_{k: 0<t_{k}<t-\epsilon \epsilon} \prod_{i: t_{k} \leq t_{i}<t-\epsilon}\left(1-N_{i}\right) \int_{t_{k-1}}^{t_{k}} S(t-s) f_{1}\left(s, v_{n-1}(s)\right) d s \\
& +\int_{t_{\bar{j}}}^{t-\epsilon} S(t-s) f_{1}\left(s, v_{n-1}(s)\right) d s \\
& +\sum_{k: 0<t_{k}<t_{\bar{j}}} \prod_{i: t_{k}<t_{i}<t-\epsilon}\left(1-N_{i}\right) S\left(t-t_{k}\right)\left(I_{k}\left(v_{n-1}\left(t_{k}\right)\right)+N_{k} v_{n-1}\left(t_{k}\right)\right) \\
& +S\left(t-t_{\bar{j}}\right)\left(I_{\bar{j}}\left(v_{n-1}\left(t_{\bar{j}}\right)\right)+N_{\bar{j}} v_{n-1}\left(t_{\bar{j}}\right)\right) \\
= & S(\epsilon)\left[\sum_{k: 0<t_{k}<t-\epsilon} \prod_{i: t_{k} \leq t_{i}<t-\epsilon}\left(1-N_{i}\right) \int_{t_{k-1}}^{t_{k}} S(t-\epsilon-s) f_{1}\left(s, v_{n-1}(s)\right) d s\right.
\end{aligned}
$$




$$
\begin{aligned}
& +\int_{t_{\bar{j}}}^{t-\epsilon} S(t-\epsilon-s) f_{1}\left(s, v_{n-1}(s)\right) d s \\
& +\sum_{k: 0<t_{k}<t_{j}} \prod_{i: t_{k}<t_{i}<t-\epsilon}\left(1-N_{i}\right) S\left(t-\epsilon-t_{k}\right)\left(I_{k}\left(v_{n-1}\left(t_{k}\right)\right)+N_{k} v_{n-1}\left(t_{k}\right)\right) \\
& \left.+S\left(t-\epsilon-t_{\bar{j}}\right)\left(I_{\bar{j}}\left(v_{n-1}\left(t_{\bar{j}}\right)\right)+N_{\bar{j}} v_{n-1}\left(t_{\bar{j}}\right)\right)\right] .
\end{aligned}
$$

By assumption $\left(\mathrm{P}_{1}\right)$ we know that

$$
f\left(t, v_{0}(t)\right)+C v_{0}(t) \leq f\left(t, v_{n-1}(t)\right)+C v_{n-1}(t) \leq f\left(t, w_{0}(t)\right)+C w_{0}(t) .
$$

Since $f\left(t, v_{0}(t)\right)$ and $f\left(t, w_{0}(t)\right)$ are continuous in the compact set $[0, \omega]$, so their image sets are compact sets in $X$, namely image sets are bounded. Combining this fact with the normality of cone $K$ in $X$, we have $\exists M_{1}>0, \forall v_{n-1} \in G_{0}$,

$$
\left\|f_{1}\left(t, v_{n-1}(t)\right)\right\| \leq\left\|f_{1}\left(t, v_{0}(t)\right)\right\|+N_{0}\left\|f_{1}\left(t, w_{0}(t)\right)-f_{1}\left(t, v_{0}(t)\right)\right\| \leq M_{1} .
$$

By assumption $\left(\mathrm{P}_{2}\right)$ we know that

$$
\begin{aligned}
I_{k}\left(v_{0}\left(t_{k}\right)\right)+N_{k} v_{0}\left(t_{k}\right) & \leq I_{k}\left(v_{n-1}\left(t_{k}\right)\right)+N_{k} v_{n-1}\left(t_{k}\right) \\
& \leq I_{k}\left(w_{0}\left(t_{k}\right)\right)+N_{k} w_{0}\left(t_{k}\right), \quad k=1,2, \ldots, p .
\end{aligned}
$$

By the normality of cone $K$ in $X$, there exists $M_{2}>0$ such that

$$
\begin{aligned}
& \left\|I_{k}\left(v_{n-1}\left(t_{k}\right)\right)+N_{k} v_{n-1}\left(t_{k}\right)\right\| \\
& \quad \leq\left\|I_{k}\left(v_{0}\left(t_{k}\right)\right)+N_{k} v_{0}\left(t_{k}\right)\right\|+N_{0}\left\|I_{k}\left(w_{0}\left(t_{k}\right)\right)-I_{k}\left(v_{0}\left(t_{k}\right)\right)+N_{k} w_{0}\left(t_{k}\right)-N_{k} v_{0}\left(t_{k}\right)\right\| \\
& \quad \leq M_{2} .
\end{aligned}
$$

Combining (4.12) with the compactness of $S(\epsilon)$, then $Y_{\epsilon}(t)=\left\{W_{\epsilon}\left(v_{n-1}\right)(t) \mid v_{n-1} \in G_{0}\right\}$ is a relatively compact set in $X$. Let $J_{1}=\left[t_{0}, t_{1}\right], J_{k}=\left(t_{k-1}, t_{k}\right], k=2,3, \ldots, p+1$, where $t_{0}=0$, $t_{p+1}=\omega$. For sufficiently small $\epsilon$ and $t, t-\epsilon \in J_{k}(k=1,2, \ldots, p+1)$, then $j=\bar{j}$ and

$$
\begin{aligned}
& \left\|W\left(v_{n-1}\right)(t)-W_{\epsilon}\left(v_{n-1}\right)(t)\right\| \\
& \quad=\left\|\int_{t_{j}}^{t} S(t-s) f_{1}\left(s, v_{n-1}(s)\right) d s-\int_{t_{j}}^{t-\epsilon} S(t-s) f_{1}\left(s, v_{n-1}(s)\right) d s\right\| \\
& \quad \leq \int_{t-\epsilon}^{t}\|S(t-s)\|\left\|f_{1}\left(s, v_{n-1}(s)\right)\right\| d s \leq M M_{1} \epsilon,
\end{aligned}
$$

hence $Y(t)$ is a totally bounded set in $X$, thus it is a relatively compact set. Especially, by the compactness of $Y(\omega)$ and the relative compactness of $\left\{B_{2}\left(v_{n-1}\right) \mid v_{n-1} \in G_{0}\right\}=(I-$ $\left.\prod_{k=1}^{p}\left(1-N_{k}\right) S(\omega)\right)^{-1} Y(\omega)$ in $X$, we know that $\left\{\left(\prod_{k: 0<t_{k}<t}\left(1-N_{k}\right)\right) S(t) B_{2}\left(v_{n-1}\right) \mid v_{n-1} \in G_{0}\right\}$ is a relatively compact set.

Noticing

$$
\left\{Q_{2}(t) \mid v_{n-1} \in G_{0}\right\}=\left\{\prod_{k: 0<t_{k}<t}\left(1-N_{k}\right) S(t) B_{2}\left(v_{n-1}\right)+W\left(v_{n-1}\right)(t) \mid v_{n-1} \in G_{0}\right\},
$$


and $Q_{2}\left(v_{n-1}\right)(0)=B_{2}\left(v_{n-1}\right)$, considering

$$
\begin{aligned}
Q_{2}\left(v_{n-1}\right)(\omega)= & {\left[\prod_{k=1}^{p}\left(1-N_{k}\right) S(\omega)\left(I-\prod_{k=1}^{p}\left(1-N_{k}\right) S(\omega)\right)^{-1}+I\right] } \\
& \times\left[\sum_{k=1}^{p} \prod_{i=k}^{p}\left(1-N_{i}\right) \int_{t_{k-1}}^{t_{k}} S(\omega-s) f_{1}\left(s, v_{n-1}(s)\right) d s\right. \\
& +\int_{t_{p}}^{\omega} S(\omega-s) f_{1}\left(s, v_{n-1}(s)\right) d s \\
& +\sum_{k=1}^{p-1} \prod_{i=k+1}^{p}\left(1-N_{i}\right) S\left(\omega-t_{k}\right)\left(I_{k}\left(v_{n-1}\left(t_{k}\right)\right)+N_{k} v_{n-1}\left(t_{k}\right)\right) \\
& \left.+S\left(\omega-t_{p}\right)\left(I_{p}\left(v_{n-1}\left(t_{p}\right)\right)+N_{p} v_{n-1}\left(t_{p}\right)\right)\right] \\
= & B_{2}\left(v_{n-1}\right),
\end{aligned}
$$

then $Q_{2}\left(v_{n-1}\right)(0)=Q_{2}\left(v_{n-1}\right)(\omega)=B_{2}\left(v_{n-1}\right)$, namely $\left\{Q_{2}\left(v_{n-1}\right)(0) \mid v_{n-1} \in G_{0}\right\}=B_{2}\left(G_{0}\right)$ is relatively compact.

Therefore, $\left\{v_{n}(t)\right\}=\left\{Q_{2}\left(v_{n-1}\right)(t) \mid v_{n-1} \in G_{0}, t \in J\right\}$ is relatively compact in $X$. Combining this fact with the monotonicity of $\left\{v_{n}\right\}$, we easily prove that $\left\{v_{n}(t)\right\}$ is convergent. Let $\left\{v_{n}(t)\right\} \rightarrow \underline{u}(t)$ in $t \in J$.

The same idea can be used to prove that $\left\{w_{n}(t)\right\} \rightarrow \bar{u}(t)$ in $t \in J$.

Evidently $\left\{v_{n}(t)\right\},\left\{w_{n}(t)\right\} \in P C(J, X)$, so $\underline{u}(t)$ and $\bar{u}(t)$ are bounded integrable in $J_{k}(k=$ $1,2, \ldots, p)$. Since for any $t \in J_{k}, v_{n}(t)=Q_{2}\left(v_{n-1}\right)(t), w_{n}(t)=Q_{2}\left(w_{n-1}\right)(t)$, letting $n \rightarrow \infty$, by the Lebesgue dominated convergence theorem, we have $\underline{u}(t)=Q_{2}(\underline{u})(t), \bar{u}(t)=Q_{2}(\bar{u})(t)$ and $\underline{u}(t), \bar{u}(t) \in P C(J, X)$. Combining this with monotonicity (4.10), we have $v_{0}(t) \leq \underline{u}(t) \leq$ $\bar{u}(t) \leq w_{0}(t)$.

Next, we prove that $\underline{u}(t)$ and $\bar{u}(t)$ are the minimal and maximal fixed points of $Q_{2}$ in $\left[v_{0}, w_{0}\right]$, respectively. In fact, for any $u^{*} \in\left[v_{0}, w_{0}\right], Q_{2}\left(u^{*}\right)=u^{*}$, we have $v_{0} \leq u^{*} \leq w_{0}$, and $v_{1}=Q_{2}\left(v_{0}\right) \leq Q_{2}\left(u^{*}\right)=u^{*} \leq Q_{2}\left(w_{0}\right)=w_{1}$. Continuing such progress, we get $v_{n} \leq u^{*} \leq w_{n}$. Letting $n \rightarrow \infty$, we get $\underline{u}(t) \leq u^{*} \leq \bar{u}(t)$. Therefore, $\underline{u}(t)$ and $\bar{u}(t)$ are the minimal and maximal $\omega$-periodic mild solutions of IPBVP (1.2) between $v_{0}$ and $w_{0}$, which can be obtained by monotone iterative sequences starting from $v_{0}$ and $w_{0}$, respectively. This completes the proof of Theorem 4.1.

Remark 4.2 In [15], the impulsive functions are required to be ordered increasing; therefore, Theorem 4.1 in this paper extensively generalizes the main results in [15].

Theorem 4.3 Let $X$ be an ordered Banach space whose positive cone $K$ is regular, $A$ : $D(A) \subset X \rightarrow X$ be a closed linear operator and $-A$ generate a positive $C_{0}$-semigroup $T(t)$ $(t \geq 0)$ in $X . f \in C(J \times X, X)$ and $f$ is $\omega$-periodic about $t, I_{k} \in C(X, X), k=1,2, \ldots, p$. Assume that IPBVP (1.2) has coupled lower and upper solutions $v_{0}$ and $w_{0}$ with $v_{0}(t) \leq w_{0}(t)(t \in J)$, and conditions $\left(\mathrm{P}_{1}\right)$ and $\left(\mathrm{P}_{2}\right)$ are satisfied, then IPBVP (1.2) has minimal and maximal $\omega$-periodic mild solutions $\underline{u}$ and $\bar{u}$ between $v_{0}$ and $w_{0}$, which can be obtained by monotone iterative sequences starting from $v_{0}$ and $w_{0}$. 
Proof From Theorem 4.1 we know that $Q_{2}:\left[v_{0}, w_{0}\right] \rightarrow\left[v_{0}, w_{0}\right]$ is a continuously increasing operator. Similarly, the two sequences $\left\{v_{n}(t)\right\}$ and $\left\{w_{n}(t)\right\}$ are defined in $\left[v_{0}, w_{0}\right]$ by the iterative scheme (4.9). By conditions $\left(\mathrm{P}_{2}\right)$, then $\left\{v_{n}(t)\right\}$ and $\left\{w_{n}(t)\right\}$ are ordered-monotonic and ordered-bounded sequences in $X$.

Using the regularity of the cone $K$, any ordered-monotonic and ordered-bounded sequence in $X$ is convergent. So, $\left\{v_{n}(t)\right\}$ and $\left\{w_{n}(t)\right\}$ are convergent, namely $\exists v^{*}(t), w^{*}(t)$, $v_{n}(t) \rightarrow v^{*}(t), w_{n}(t) \rightarrow w^{*}(t)$ in $t \in J^{\prime}$, where $v^{*}(t), w^{*}(t)$ are bounded and strongly measurable. Combining (4.3) with $v_{n}=Q_{2}\left(v_{n-1}\right)$, let $C>v_{0}$, noticing $S(t)=e^{-C t} T(t)(t \geq 0)$ is an exponentially stable and positive $C_{0}$-semigroup in $X$, letting $n \rightarrow \infty$, by the Lebesgue dominated convergence theorem, we have $v^{*}(t)=Q_{2}\left(v^{*}\right)(t) \in P C(J, X)$.

Similarly, we prove that $w^{*}(t) \in P C(J, X)$ and $w^{*}(t)=Q_{2}\left(w^{*}\right)(t)$.

By (4.10), we know $v_{0}(t) \leq v^{*}(t) \leq w^{*}(t) \leq w_{0}(t)$.

Similar to the proof of Theorem 4.1, we know that $\underline{u}(t)$ and $\bar{u}(t)$ are the minimal and maximal $\omega$-periodic mild solutions of IPBVP (1.2) between $v_{0}$ and $w_{0}$, which can be obtained by monotone iterative sequences starting from $v_{0}$ and $w_{0}$, respectively. This completes the proof of Theorem 4.3.

Corollary 4.4 Let $X$ be an ordered and weakly sequentially complete Banach space, whose positive cone $K$ is normal, $A: D(A) \subset X \rightarrow X$ be a closed linear operator and $-A$ generate a positive $C_{0}$-semigroup $T(t)(t \geq 0)$ in $X . f \in C(J \times X, X)$ and $f$ is $\omega$-periodic about $t$, $I_{k} \in C(X, X), k=1,2, \ldots, p$. Assume that IPBVP (1.2) has lower and upper solutions $v_{0}$ and $w_{0}$ with $v_{0}(t) \leq w_{0}(t)(t \in J)$, and conditions $\left(\mathrm{P}_{1}\right)$ and $\left(\mathrm{P}_{2}\right)$ are satisfied, then IPBVP (1.2) has minimal and maximal $\omega$-periodic mild solutions $\underline{u}$ and $\bar{u}$ between $v_{0}$ and $w_{0}$, which can be obtained by monotone iterative sequences starting from $v_{0}$ and $w_{0}$.

Proof In an ordered and weakly sequentially complete Banach space, the normal cone $K$ is regular. Then the proof is complete.

Next, we discuss the existence of the $\omega$-periodic mild solutions of IPBVP (1.2), when the lower and upper solutions of IPBVP (1.2) do not exist.

Theorem 4.5 Let $X$ be an ordered Banach space, whose positive cone $K$ is normal, $A$ : $D(A) \subset X \rightarrow X$ be a closed linear operator and $-A$ generate an exponentially stable, compact and positive $C_{0}$-semigroup $T(t)(t \geq 0)$ in $X . f \in C(J \times X, X)$ and $f$ is $\omega$-periodic about $t, I_{k} \in C(X, X), k=1,2, \ldots, p$ satisfy $\left(\mathrm{P}_{1}\right)$ and $\left(\mathrm{P}_{2}\right)$ and the following conditions:

$\left(\mathrm{P}_{3}\right) \exists 0<a<-v_{0}\left(v_{0}\right.$ is the growth index of $\left.T(t)\right), h \in P C(J, X), h \geq \theta$, such that

$$
-a x-h(t) \leq f(t,-x), \quad f(t, x) \leq a x+h(t) .
$$

$\left(\mathrm{P}_{4}\right)$ Let $a_{k}<1, \frac{1}{\omega} \sum_{k=1}^{p} \ln \left(1-a_{k}\right)<-a-v_{0}, e_{k} \geq \theta$, such that

$$
a_{k} x-e_{k} \leq I_{k}(-x), \quad I_{k}(x) \leq-a_{k} x+e_{k} .
$$

Then IPBVP (1.2) has minimal and maximal $\omega$-periodic mild solutions, which can be obtained by monotone iterative sequences. 
Proof For $0<a<-v_{0}$, then $-(A-a I)$ generates an exponentially stable and positive $C_{0}$-semigroup $e^{a t} T(t)(t \geq 0)$, whose growth index is $a+v_{0}$. For $\frac{1}{\omega} \sum_{k=1}^{p} \ln \left(1-a_{k}\right)<-a-v_{0}$, $h(t) \geq \theta, e_{k} \geq \theta$, by Theorem 3.1, then the periodic boundary value problem of linear impulsive evolution equation (LIPBVP) in $X$,

$$
\left\{\begin{array}{l}
u^{\prime}(t)+A u(t)-a u(t)=h(t), \quad t \in J, t \neq t_{k} \\
\left.\Delta u\right|_{t=t_{k}}+a_{k} u\left(t_{k}\right)=e_{k}, \quad k=1,2, \ldots, p \\
u(0)=u(\omega)
\end{array}\right.
$$

has a unique positive solution $u^{*} \geq \theta$. Let $v_{0}=-u^{*}, w_{0}=u^{*}$, by conditions $\left(\mathrm{P}_{1}\right),\left(\mathrm{P}_{2}\right),\left(\mathrm{P}_{3}\right)$ and $\left(\mathrm{P}_{4}\right)$, we get

$$
\left\{\begin{array}{l}
v_{0}^{\prime}(t)+A v_{0}(t)=a v_{0}(t)-h(t) \leq f\left(t, v_{0}(t)\right), \quad t \in J, t \neq t_{k}, \\
\left.\Delta v_{0}\right|_{t=t_{k}}=-a_{k} v_{0}\left(t_{k}\right)-e_{k} \leq I_{k}\left(v_{0}\left(t_{k}\right)\right), \quad k=1,2, \ldots, p, \\
u(0) \leq u(\omega)
\end{array}\right.
$$

and

$$
\left\{\begin{array}{l}
w_{0}^{\prime}(t)+A w_{0}(t)=a w_{0}(t)+h(t) \geq f\left(t, w_{0}(t)\right), \quad t \in J, t \neq t_{k}, \\
\left.\Delta w_{0}\right|_{t=t_{k}}=-a_{k} w_{0}\left(t_{k}\right)+e_{k} \geq I_{k}\left(w_{0}\left(t_{k}\right)\right), \quad k=1,2, \ldots, p, \\
u(0) \geq u(\omega) .
\end{array}\right.
$$

So, we showed that $v_{0}$ and $w_{0}$ are a lower solution and an upper solution of IPBVP (1.2). By Theorem 4.1, our conclusion holds. Then the proof is complete.

Corollary 4.6 Let $X$ be an ordered Banach space, whose positive cone $K$ is regular, $A$ : $D(A) \subset X \rightarrow X$ be a closed linear operator and $-A$ generate a positive $C_{0}$-semigroup $T(t)$ $(t \geq 0)$ in $X . f \in C(J \times X, X)$ and $f$ is $\omega$-periodic about $t, I_{k} \in C(X, X), k=1,2, \ldots, p$. If conditions $\left(\mathrm{P}_{1}\right),\left(\mathrm{P}_{2}\right),\left(\mathrm{P}_{3}\right)$ and $\left(\mathrm{P}_{4}\right)$ are satisfied, then IPBVP $(1.2)$ has minimal and maximal $\omega$-periodic mild solutions, which can be obtained by monotone iterative sequences.

Corollary 4.7 Let $X$ be an ordered and weakly sequentially complete Banach space, whose positive cone $K$ is normal, $A: D(A) \subset X \rightarrow X$ be a closed linear operator and $-A$ generate a positive $C_{0}$-semigroup $T(t)(t \geq 0)$ in $X . f \in C(J \times X, X)$ and $f$ is $\omega$-periodic about $t, I_{k} \in$ $C(X, X), k=1,2, \ldots, p$. If conditions $\left(\mathrm{P}_{1}\right),\left(\mathrm{P}_{2}\right),\left(\mathrm{P}_{3}\right)$ and $\left(\mathrm{P}_{4}\right)$ are satisfied, then IPBVP (1.2) has minimal and maximal $\omega$-periodic mild solutions, which can be obtained by monotone iterative sequences.

\section{Example}

Example 5.1 In order to apply our results, we consider the following impulsive parabolic partial differential equation:

$$
\left\{\begin{array}{l}
\frac{\partial}{\partial t} u(x, t)+A(x, D) u(x, t)=f(x, t, u(x, t)), \quad x \in \Omega, t \in J, t \neq t_{k}, \\
\left.\Delta u\right|_{t=t_{k}}=I_{k}\left(u\left(x, t_{k}\right)\right), \quad x \in \Omega, k=1,2, \ldots, m, \\
B u=0, \quad(x, t) \in \partial \Omega \times J \\
u(x, 0)=u(x, \omega)
\end{array}\right.
$$


where $J=[0, \omega], 0<t_{1}<t_{2}<\cdots<t_{m}<\omega, J^{\prime}=J \backslash\left\{t_{1}, t_{2}, \ldots, t_{m}\right\}, J^{\prime \prime}=J \backslash\left\{0, t_{1}, t_{2}, \ldots, t_{m}\right\}$, integer $N>1, \Omega \in \mathbf{R}^{N}$ is a bounded domain with a sufficiently smooth boundary $\partial \Omega$,

$$
A(x, D)=-\sum_{i=1}^{N} \sum_{j=1}^{N} a_{i j}(x) \frac{\partial^{2}}{\partial x_{i} \partial y_{j}}+\sum_{i=1}^{N} a_{i}(x) \frac{\partial}{\partial x_{i}}+a_{0}(x)
$$

is a strongly elliptic operator of second order, coefficient functions $a_{i j}(x), a_{i}(x)$ and $a_{0}(x)$ are Hölder continuous in $\Omega, B u=b_{0}(x) u+\delta \frac{\partial u}{\partial n}$ is a regular boundary operator on $\partial \Omega$, $f: \bar{\Omega} \times J \times \mathbf{R} \rightarrow \mathbf{R}$ is continuous, $I_{k}: \mathbf{R} \rightarrow \mathbf{R}$ are also continuous, $k=1,2, \ldots, m$.

Let $X=L^{p}(\Omega)$ with $p>N+2, K=\left\{u \in L^{p}(\Omega) \mid u(x) \geq 0\right.$ a.e. $\left.x \in \Omega\right\}$, and define the operator $A$ as follows:

$$
D(A)=\left\{u \in W^{2, p}(\Omega) \mid B u=0\right\}, \quad A u=A(x, D) u .
$$

We know that $X$ is a Banach space, $K$ is a regular cone of $X$, and $-A$ generates a positive and analytic $C_{0}$-semigroup $T(t)(t \geq 0)$ in $X$ (see [29]). Define $u(t)=u(\cdot, t), f(t, u(t))=$ $f(\cdot, t, u(\cdot, t)), I_{k}\left(u\left(t_{k}\right)\right)=I_{k}\left(u\left(\cdot, t_{k}\right)\right)$, then system (5.1) can be reformulated as IPBVP (1.2) in $X$. We assume that the following conditions hold:

(i) Let $f(x, t, 0) \geq 0,, I_{k}(0) \geq 0, u(x, \omega) \geq u(x, 0) \geq 0, x \in \Omega$.

(ii) There exist $w=w(x, t) \in P C(J, X) \cap C^{2,1}$ and $w(x, t) \geq 0, x \in \Omega, t \in J_{k}$ such that

$$
\left\{\begin{array}{l}
\frac{\partial w}{\partial t}+A(x, D) w \geq f(x, t, w), \quad x \in \Omega, t \in J, t \neq t_{k}, \\
\left.\Delta w\right|_{t=t_{k}} \geq I_{k}\left(w\left(x, t_{k}\right)\right), \quad x \in \Omega, k=1,2, \ldots, m, \\
B w=0, \quad(x, t) \in \partial \Omega \times J \\
w(x, 0) \geq w(x, \omega)
\end{array}\right.
$$

(iii) The partial derivative $f_{u}^{\prime}(x, t, u)$ is continuous on any bounded domain.

(iv) For any $u_{1}, u_{2} \in[0, w(x, t)]$ with $u_{1} \leq u_{2}$, there exists $0<N_{k}<1$; for any $x \in \Omega$, $k=1,2, \ldots, m$, we have

$$
I_{k}\left(u_{2}\left(x, t_{k}\right)\right)-I_{k}\left(u_{1}\left(x, t_{k}\right)\right) \geq-N_{k}\left(u_{2}\left(x, t_{k}\right)-u_{1}\left(x, t_{k}\right)\right) .
$$

Theorem 5.2 If assumptions (i), (ii), (iii) and (iv) are satisfied, then the impulsive parabolic partial differential equation (5.1) has minimal and maximal mild solutions between 0 and $w(x, t)$, which can be obtained by a monotone iterative procedure starting from 0 and $w(x, t)$, respectively.

Proof From assumptions (i) and (ii) we know that 0 and $w(x, t)$ are lower and upper solutions of IBPVP (5.1), respectively. (iii) implies that condition $\left(\mathrm{P}_{1}\right)$ is satisfied. (iv) implies that condition $\left(\mathrm{P}_{2}\right)$ is satisfied. So, by Theorem 4.3, we have the result. Then the proof is complete.

\section{Conclusions}

In this paper, we have discussed the existence of $\omega$-periodic mild solutions for the impulsive evolution equation by means of the perturbation method and the mixed monotone iterative technique under the impulsive functions satisfying quasimonotonicity. The main result (Theorem 4.1) is new and the following results appear as its special cases: 
(i) If we take $A=0$ in (1.2), we obtain the results for first-order periodic boundary problem for impulsive ordinary differential equations.

(ii) If $I_{k}\left(u\left(t_{k}\right)\right)=0, k=1,2, \ldots, p$, in (1.2), then Theorem 4.1 in this paper is Theorem 3.1 in [30].

(iii) If $N_{k}=0$ in condition $\left(\mathrm{P}_{2}\right)$, then Theorem 4.1 in this paper is Theorem 1 in [27].

\section{Competing interests}

The authors declare that they have no competing interests.

\section{Authors' contributions}

Each of the authors contributed to each part of this study equally and approved the final version of this manuscript.

\section{Acknowledgements}

The authors are very grateful to the editor and the reviewers for their constructive comments and suggestions. This work is supported by NNSF of China (11261053, 11401473, 11501455), NSF of Gansu Province (1208RJZA129) and the Fundamental Research Funds for the Central Universities (31920130010).

Received: 30 June 2015 Accepted: 30 September 2015 Published online: 19 October 2015

\section{References}

1. Lakshmikantham, V, Bainov, DD, Simeonov, PS: Theory of Impulsive Differential Equations. World Scientific, Singapore (1989)

2. Benchohra, M, Henderson, J, Ntouyas, S: Impulsive Differential Equations and Inclusions. Contemp. Math. Appl., vol. 2 (2006)

3. Chen, P: Mixed monotone iterative technique for impulsive periodic boundary value problems in Banach spaces. Bound. Value Probl. 2011, Article ID 421261 (2011)

4. Li, Y, Liu, Z: Monotone iterative technique for addressing impulsive integro-differential equations in Banach spaces. Nonlinear Anal. 66, 83-92 (2007)

5. Yang, $\mathrm{H}$ : Mixed monotone iterative technique for abstract impulsive evolution equations in Banach spaces. J. Inequal. Appl. 2010, Article ID 293410 (2010)

6. Lan, HY: Monotone method for a system of nonlinear mixed type implicit impulsive integro-differential equations in Banach spaces. Comput. Math. Appl. 222, 531-543 (2008)

7. Ahmad, B, Alsaedi, A: Existence of solutions for anti-periodic boundary value problems of nonlinear impulsive functional integro-differential equations of mixed type. Nonlinear Anal. 3(4), 501-509 (2009)

8. Liang, J, Liu, JH, Xiao, TJ: Nonlocal impulsive problems for nonlinear differential equations in Banach spaces. Math. Comput. Model. 49, 798-804 (2009)

9. Liang, J, Liu, JH, Xiao, T: Periodic solutions of delay impulsive differential equations. Nonlinear Anal. 74, 6835-6842 (2011)

10. Wang, JR, Xiang, X, Peng, Y: Periodic solutions of semilinear impulsive periodic system on Banach space. Nonlinear Anal., Theory Methods Appl. 71, e1344-e1353 (2009)

11. Ahmed, NU: Impulsive evolution equations in infinite dimensional spaces. Dyn. Contin. Discrete Impuls. Syst., Ser. A Math. Anal. 10, 11-24 (2003)

12. Peng, $Y, X i a n g, X$, Jiang, $Y$ : A class of semilinear evolution equations with impulses at variable times on Banach spaces. Nonlinear Anal. 11, 3984-3992 (2010)

13. Luo, ZG, Nieto, JJ: New results for the periodic boundary value problem for impulsive integro-differential equations. Nonlinear Anal. 70, 2248-2260 (2009)

14. Luo, Z, Jing, Z: Periodic boundary value problem for first-order impulsive functional differential equations. Comput. Math. Appl. 55, 2094-2107 (2008)

15. Luo, Z, Shen, J, Nieto, JJ: Antiperiodic boundary value problem for first-order impulsive ordinary differential equation. Comput. Math. Appl. 49, 253-261 (2005)

16. Chen, $\mathrm{P}, \mathrm{Li}, \mathrm{Y}, \mathrm{Yang}, \mathrm{H}$ : Perturbation method for nonlocal impulsive evolution equations. Nonlinear Anal. 8, 22-30 (2013)

17. Hernández M, E, Tanaka Aki, SM: Global solutions for abstract impulsive differential equations. Nonlinear Anal. 72 , 1280-1290 (2010)

18. Wang, JR, Zhou, Y, Fečkan, M: Nonlinear impulsive problems for fractional differential equations and Ulam stability. Comput. Math. Appl. 64, 3389-3405 (2012)

19. Guo, TL, Zhang, KJ: Impulsive fractional partial differential equations. Appl. Math. Comput. 257, 581-590 (2015)

20. Du, S, Lakshmikantham, V: Monotone iterative technique for differential equations in Banach spaces. J. Math. Anal. Appl. 87, 454-459 (1982)

21. Sun, J, Zhao, Z: Extremal solutions of initial value problem for integro-differential equations of mixed type in Banach spaces. Ann. Differ. Equ. 8, 469-475 (1992)

22. Guo, D, Liu, X: Extremal solutions of nonlinear impulsive integro differential equations in Banach spaces. J. Math. Anal. Appl. 177, 538-552 (1993)

23. Wang, L, Wang, Z: Monotone iterative techniques for parameterized BVPs of abstract semilinear evolution equations. Comput. Math. Appl. 46, 1229-1243 (2003)

24. Chen, $\mathrm{P}, \mathrm{Mu}, \mathrm{J}$ : Monotone iterative method for semilinear impulsive evolution equations of mixed type in Banach spaces. Electron. J. Differ. Equ. 2010, 149 (2010)

25. Chen, $\mathrm{P}, \mathrm{Li}, \mathrm{Y}$ : Mixed monotone iterative technique for a class of semilinear impulsive evolution equations in Banach spaces. Nonlinear Anal. 74, 3578-3588 (2011) 
26. Ahmad, B, Nieto, Jj: Existence and approximation of solutions for a class of nonlinear impulsive functional differential equations with anti-periodic boundary conditions. Nonlinear Anal. 69, 3291-3298 (2008)

27. Shao, YB, Zhang, HH: Monotone iterative technique of periodic solutions for impulsive evolution equations in Banach space. J. Comput. Anal. Appl. 17, 48-58 (2014)

28. Li, YX: Existence and uniqueness of positive periodic solutions for abstract semilinear evolution equations. J. Syst. Sci. Math. Sci. 25(6), 720-728 (2005) (in Chinese)

29. Pazy, A: Semigroups of Linear Operators and Applications to Partial Differential Equations. Springer, New York (1983)

30. Li, YX: Periodic solutions of semilinear evolution equations in Banach spaces. Acta Math. Sin. 41(3), 629-636 (1998) (in Chinese)

Submit your manuscript to a SpringerOpen ${ }^{\odot}$ journal and benefit from:

- Convenient online submission

- Rigorous peer review

- Immediate publication on acceptance

- Open access: articles freely available online

- High visibility within the field

- Retaining the copyright to your article 